\title{
La formación de docentes de lenguas extranjeras en la Argentina: aportes para la construcción de un espacio de debate necesario
}

\author{
GUSTAVO MÓRTOLA ${ }^{(1)}$ \\ MARIANO MONTSERRAT ${ }^{(2)}$
}

Fecha de recepción 29/05/18

Fecha de aceptación: 01/09/18

Resumen. En este artículo realizaremos una descripción cuantitativa del sistema de formación docente terciario en lenguas extranjeras a nivel nacional con el propósito de mapear su configuración actual y dar cuenta de algunas de sus características específicas. Con este propósito, presentaremos algunos datos de tipo cuantitativos tales como el número de profesorados, su presencia diferenciada en las distintas jurisdicciones, el tipo de oferta con la que cuentan, guarismos vinculados con los estudiantes, entre otros. Además, ofreceremos algunas coordenadas interpretativas para explicar la hegemonía del inglés en este campo en un tiempo en que las perspectivas plurilingües priman en el mundo académico de las lenguas extranjeras.

Palabras clave. políticas lingüísticas . formación docente $\cdot$ lenguas extranjeras . hegemonía del inglés · plurilingüismo

(1) Profesor para la Enseñanza Primaria. Lic. en Ciencias de la Educación (UBA). Magíster en Política y Administración de la Educación (UnTref). Responsable del Área de Formación Docente de la Universidad Nacional Arturo Jauretche. Docente de la asignatura «Trabajo docente» «ENS N ${ }^{\circ} 3$ Bernardino Rivadavia» - CABA) y del "Taller de formación docente» (Licenciatura de Kinesiología y Fisiatría - UNAJ).

formaciondocente.unaj@ gmail.com
(2) Profesor de Inglés egresado del IsP Joaquín V. González, Magíster en Educación por la Universidad de San Andrés y Doctorando en Educación por la Universidad de San Andrés. Docente de Lengua Inglesa en el Isp Joaquín V González y el Isp en Lenguas Vivas Juan Ramón Fernández. Docente de comprensión lectora en inglés de la Universidad Nacional Arturo Jauretche. marianmont2868@gmail.com 


\section{Foreign Language Teacher Training at College Level in Argentina: contributions to the construction of a necessary debate}

\begin{abstract}
This article presents a quantitative description of the foreign language teacher education system at college in Argentina with the aim of mapping its current configuration and giving an account of some of its specific characteristics. For this purpose, we will present some quantitative data such as the number of teacher training colleges, their differentiated presence in the various jurisdictions, offered education, and statistics related to students, among others. We will also
\end{abstract}

provide some interpretative coordinates to explain the hegemony of English in this field at a time when plurilingual perspectives prevail in the academic world of foreign languages.

\footnotetext{
Keywords. políticas lingüísticas . formación docente lenguas extranjeras . hegemonía del inglés · plurilingüismo
}

\section{Introducción}

La enseñanza de las lenguas extranjeras y la formación de docentes para tal tarea cuentan con una larga tradición en la Argentina. En el sistema educativo se puede señalar que tanto el inglés como el francés, el alemán, el italiano y más recientemente el portugués, han pugnado por consolidar un espacio en el curriculum y disputar espacios por la constitución de carreras de profesorado tanto en las universidades como en el sistema superior no universitario.

Esta reflexión inicial no es más que una de las posibles vías de acceso para aproximarnos al que será el eje vertebrador de este artículo: un análisis cuantitativo del sistema terciario de formación de docentes en lenguas extranjeras en nuestro país. Para ello presentaremos un mapeo de base cuantitativa a través del cual indagaremos diversas cuestiones tales como las instituciones existentes, su distribución en las provincias, las relaciones entre las lenguas extranjeras, los estudiantes y el problema general de la terminalidad en este campo. Repetimos: se trata de un artículo cuyo objetivo es el de presentar datos y discutirlos a la luz de comprender un sistema ya conformado y las lógicas que han conducido a tal conformación. Es aquí donde radica nuestro interés y preocupación centrales, ya que difícilmente puedan pensarse políticas efectivas 
en el futuro si se desconoce el territorio real en el que estas están llamadas a producir efectos. No partimos del vacío para realizar el abordaje estadístico que presentamos, pues existen varios trabajos desde la década del noventa al presente que intentaron «fotografiar» el sistema formador de docentes en nuestro país y se han constituido en referencias ineludibles de nuestra indagación (Diker y Terigi, 1994, Davini, 2005, Aguerrondo y Vezub, 2011, INFD, 2014). Sin embargo, no encontramos antecedentes que hayan dado cuenta de la situación de la formación de docentes de lenguas extranjeras con base cuantitativa que haga foco en el sistema terciario.

Compartiremos entonces a continuación algunos datos de dicho sistema para analizar su conformación a la búsqueda de sus regularidades y caracteres predecibles.

\section{Algunas características del sistema formador de docentes de lenguas extranjeras}

\section{¿Una oferta plurilingüe?}

¿Cómo es la oferta de profesorados terciarios de idiomas extranjeros en nuestro país? En el año 1904, el entonces Ministro de Instrucción Pública y Justicia Juan Ramón Fernández impulsó la creación en la ciudad de Buenos Aires (CABA) de Ios Institutos de Profesorado de Lenguas Vivas y del Instituto Nacional Superior del Profesorado como seminario pedagógico. Dicha creación puso de manifiesto la necesidad de formar docentes que pudiesen enseñar idiomas extranjeros en el nivel medio, necesidad que Fernández sustentaba en que «la enseñanza se realiza en condiciones lamentables por su profesorado improvisado» (Bein, 2012). Desde ese acontecimiento seminal en la formación de docentes en lenguas extranjeras hasta la coyuntura actual, la oferta se ha extendido a lo largo y a lo ancho del territorio nacional, incorporándose también al sector privado y a la universidad, aunque estos últimos con un protagonismo marcadamente menor. La Tabla 1 expresa que en la actualidad hay 258 carreras de profesorado, de las cuales 223 se encuentran en los institutos de formación docente (IFD) y solo 35 son ofertadas por las universidades. Es notable que del total de esas carreras, $212(82,2 \%)$ son de inglés mientras que el francés con $19(7,4 \%)$ y el portugués con $19(7,4 \%)$ se ubican en un lejano segundo lugar. Si se focaliza 


\begin{tabular}{|c|c|c|c|c|c|c|c|c|c|c|}
\hline \multirow{2}{*}{$\begin{array}{l}\text { Lengua } \\
\text { extranjera }\end{array}$} & \multicolumn{2}{|c|}{ Profesorados terciarios } & \multirow{2}{*}{$\begin{array}{l}\text { Total Prof. } \\
\text { Terciarios }\end{array}$} & \multirow[t]{2}{*}{$\%$} & \multicolumn{2}{|c|}{ Profesorados universitarios } & \multirow{2}{*}{$\begin{array}{c}\text { Total Prof. } \\
\text { Universitarios }\end{array}$} & \multirow[t]{2}{*}{$\%$} & \multirow{2}{*}{$\begin{array}{c}\text { Total } \\
\text { profesorados }\end{array}$} & \multirow[t]{2}{*}{$\%$} \\
\hline & Público & Privado & & & Público & Privado & & & & \\
\hline Inglés & 119 & 74 & 193 & 86,5 & 14 & 5 & 19 & 54,3 & 212 & 82,2 \\
\hline Francés & 12 & 1 & 13 & 5,8 & 6 & 0 & 6 & 17,1 & 19 & 7,4 \\
\hline Alemán & 1 & 0 & 1 & 0,4 & 1 & 0 & 1 & 2,9 & 2 & 0,8 \\
\hline Italiano & 2 & 2 & 4 & 1,8 & 2 & 0 & 2 & 5,7 & 6 & 2,3 \\
\hline Portugués & 6 & 6 & 12 & 5,4 & 7 & 0 & 7 & 20,0 & 19 & 7,4 \\
\hline Totales & 140 & 83 & 223 & 100,0 & 30 & 5 & 35 & 100,0 & 258 & 100,0 \\
\hline
\end{tabular}

Tabla1. Profesorados de lenguas extranjeras terciarios y universitarios (año 2018).

Fuente: Elaboración propia en base a datos de la Secretaría de Políticas Universitarias, el mapa de oferta de formación de docentes del INFD y los sitios web de las instituciones. el análisis en el subsistema terciario, este ofrece 193 carreras de profesorado en inglés $(86,5 \%)$ en tanto que en francés solo hay $13(5,8 \%)$ y en portugués $12(5,4 \%)$, lo que consecuentemente da cuenta del crecimiento gradual del portugués en los últimos años, que ya disputa un segundo lugar en importancia con el francés.

El sector privado concentra casi con exclusividad su oferta en los profesorados de inglés, con la excepción de una dinámica creciente en el idioma portugués, que cuenta con seis profesorados terciarios. La lógica de la demanda del mercado parece guiar la creación de las carreras en este sector, donde el inglés es dominante y el portugués se presenta como una lengua con un ascendente dinamismo y visibilidad.

Un rasgo que ya se vislumbra en los datos presentados y que analizaremos a lo largo de este artículo es lo que denominamos la «hegemonía del inglés». Creemos que es entonces válido interrogarse acerca de las causas por las cuales este idioma es el indiscutido triunfador en la disputa por la formación de profesores terciarios de lenguas extranjeras, hecho que como hemos visto, aparece contundentemente expresado en el número de profesorados en inglés en el subsistema terciario (86,5\%).

A partir de aquí centraremos nuestro análisis en el subsistema terciario de formación docente, ya que hemos abordado al sistema universitario en otros trabajos (Mórtola y Montserrat, 2019 en prensa). ¿Cómo se distribuyen estas 223 carreras de profesorado en lenguas extranjeras en las jurisdicciones que conforman nuestro país? En la Tabla 2 se observa que todas las jurisdicciones, con la excepción de La Pampa, poseen IFD que ofrecen tal 


\begin{tabular}{|c|c|c|c|c|c|c|c|c|c|c|c|}
\hline \multirow[t]{2}{*}{ Jurisdicción } & \multicolumn{2}{|c|}{ Prof. de Inglés } & \multicolumn{2}{|c|}{ Prof. de Francés } & \multicolumn{2}{|c|}{ Prof. de Alemán } & \multicolumn{2}{|c|}{ Prof. de Italiano } & \multicolumn{2}{|c|}{ Prof. de Portugués } & \multirow[t]{2}{*}{ Tota } \\
\hline & Público & Privado & Público & Privado & Público & Privado & Público & Privado & Público & Privado & \\
\hline Buenos Aires & 46 & 26 & 1 & 0 & 0 & 0 & 0 & 0 & 1 & 2 & 76 \\
\hline Catamarca & 8 & 2 & 0 & 0 & 0 & 0 & 0 & 0 & 0 & 0 & 10 \\
\hline Chaco & 4 & 1 & 1 & 1 & 0 & 0 & 0 & 1 & 0 & 1 & 9 \\
\hline Chubut & 5 & 2 & 0 & 0 & 0 & 0 & 0 & 0 & 0 & 0 & 7 \\
\hline CABA & 3 & 5 & 2 & 0 & 1 & 0 & 1 & 0 & 2 & 1 & 15 \\
\hline Córdoba & 5 & 10 & 0 & 0 & 0 & 0 & 0 & 0 & 0 & 0 & 15 \\
\hline Corrientes & 4 & 0 & 1 & 0 & 0 & 0 & 1 & 0 & 1 & 0 & 7 \\
\hline Entre Ríos & 5 & 4 & 0 & 0 & 0 & 0 & 0 & 0 & 0 & 0 & 9 \\
\hline Formosa & 2 & 0 & 0 & 0 & 0 & 0 & 0 & 0 & 0 & 0 & 2 \\
\hline Jujuy & 7 & 0 & 1 & 0 & 0 & 0 & 0 & 0 & 1 & 0 & 9 \\
\hline La Pampa & 0 & 0 & 0 & 0 & 0 & 0 & 0 & 0 & 0 & 0 & 0 \\
\hline La Rioja & 2 & 0 & 1 & 0 & 0 & 0 & 0 & 0 & 0 & 0 & 3 \\
\hline Mendoza & 5 & 5 & 0 & 0 & 0 & 0 & 0 & 0 & 0 & 0 & 10 \\
\hline Misiones & 0 & 3 & 0 & 0 & 0 & 0 & 0 & 0 & 0 & 2 & 5 \\
\hline Neuquén & 0 & 2 & 0 & 0 & 0 & 0 & 0 & 0 & 0 & 0 & 2 \\
\hline Río Negro & 0 & 1 & 0 & 0 & 0 & 0 & 0 & 0 & 0 & 0 & 1 \\
\hline Salta & 4 & 4 & 1 & 0 & 0 & 0 & 0 & 0 & 0 & 0 & 9 \\
\hline San Juan & 1 & 1 & 1 & 0 & 0 & 0 & 0 & 0 & 0 & 0 & 3 \\
\hline San Luis & 1 & 0 & 0 & 0 & 0 & 0 & 0 & 0 & 0 & 0 & 1 \\
\hline Santa Cruz & 1 & 1 & 0 & 0 & 0 & 0 & 0 & 0 & 0 & 0 & 2 \\
\hline Santa Fe & 9 & 3 & 2 & 0 & 0 & 0 & 0 & 1 & 0 & 0 & 15 \\
\hline Santiago del Estero & 1 & 1 & 0 & 0 & 0 & 0 & 0 & 0 & 0 & 0 & 2 \\
\hline Tierra del Fuego & 2 & 0 & 0 & 0 & 0 & 0 & 0 & 0 & 0 & 0 & 2 \\
\hline Tucumán & 4 & 3 & 1 & 0 & 0 & 0 & 0 & 0 & 1 & 0 & 9 \\
\hline Total & 119 & 74 & 12 & 1 & 1 & 0 & 2 & 2 & 6 & 6 & 223 \\
\hline
\end{tabular}

Tabla2. Distribución de los profesorados terciarios de lenguas extranjeras en

tipo de formación. Es destacable que si bien La Pampa no tiene profesorados terciarios que formen docentes en lenguas extranjeras, la Universidad Nacional de La Pampa cuenta con tal tipo de formación ofreciendo solo el profesorado en lengua inglesa.

La Tabla 3 muestra con mayor claridad cómo se distribuye la formación las provincias (año 2018) Fuente: Elaboración propia en base a datos del mapa de oferta de formación de docentes del INFD y los sitios web de las instituciones. docente terciaria en lenguas extranjeras en las provincias. Hay 13 provincias - Catamarca, Chubut, Córdoba, Entre Ríos, Formosa, La Pampa, Mendoza, 


\begin{tabular}{l|l|c}
\hline Profesorados & Provincias & Total provincias \\
\hline Inglés & $\begin{array}{l}\text { Catamarca, Chubut, Córdoba, Entre Ríos, Formosa, Mendoza, } \\
\text { Neuquén, Río Negro, San Luis, Santa Cruz, Santiago del } \\
\text { Estero, Tierra del Fuego }\end{array}$ & 12 \\
\hline Inglés y Francés & La Rioja, Salta, San Juan & 3 \\
\hline Inglés y Portugués & Misiones & 1 \\
\hline Inglés, Francés y Portugués & Buenos Aires, Jujuy, Tucumán & 3 \\
\hline Inglés, Francés e Italiano & Santa Fe & 1 \\
\hline Inglés, Francés, Italiano y Portugués & Chaco, Corrientes & 2 \\
\hline Inglés, Francés, Italiano, Alemán y Portugués & CABA & 1 \\
\hline
\end{tabular}

Tabla 3. Oferta de profesorados terciarios de lenguas extranjeras en las distintas provincias (año 2018). Fuente: Elaboración propia en base a datos del mapa de oferta de formación de docentes del INFD y los sitios web de las instituciones.
Neuquén, Río Negro, San Luis, Santa Cruz, Santiago del Estero y Tierra del Fuego - que tienen solo profesorados terciarios de inglés. La provincia de Buenos Aires deviene un caso paradigmático en este sentido, ya que a pesar de sus recursos, de sus 76 profesorados terciarios en idiomas ubicados a lo largo y ancho de su extenso territorio, solo cuatro son de lenguas distintas al inglés. Finalmente, en directa contraposición a estos casos, la ciudad de Buenos Aires se presenta como una «rara avis» entre las jurisdicciones del país, ya que se constituye como el único distrito que posee profesorados de las cinco lenguas extranjeras. En otros trabajos, hemos señalado el carácter porteño-céntrico de las políticas lingüísticas en lenguas extranjeras, que frecuentemente han mirado a las demás jurisdicciones desde su propia y privilegiada realidad (Mórtola y Montserrat, 2018).

\section{¿Dónde se ofertan las carreras de profesorado de nivel terciario en lenguas extranjeras?}

¿Qué presencia tienen las carreras de profesorado en lenguas extranjeras si se considera la oferta de IFD en tanto unidades? Para responder a este interrogante hemos depurado la base de datos del INFD denominada «Mapa de la formación docente» al mes de abril del año 2018. A partir de un control de la información disponible hemos contabilizado la existencia de un total de 1294 IFD que ofrecen una o más carreras docentes y 108 anexos y 


\begin{tabular}{|c|c|c|c|c|c|c|c|c|c|}
\hline Jurisdicción & $\begin{array}{l}\text { Unidades } \\
\text { sector } \\
\text { público }\end{array}$ & $\begin{array}{l}\text { Unidades } \\
\text { públicas con } \\
\text { profesorados } \\
\text { de idiomas }\end{array}$ & $\%$ & $\begin{array}{l}\text { Unidades } \\
\text { sector } \\
\text { privado }\end{array}$ & $\begin{array}{l}\text { Unidades } \\
\text { privadas con } \\
\text { profesorados } \\
\text { de idiomas }\end{array}$ & $\%$ & $\begin{array}{c}\text { Total } \\
\text { unidades }\end{array}$ & $\begin{array}{c}\text { Total } \\
\text { unidades } \\
\text { con prof. } \\
\text { de idiomas }\end{array}$ & $\%$ \\
\hline Buenos Aires & 244 & 47 & 19,3 & 137 & 26 & 19,0 & 381 & 73 & 19,2 \\
\hline Catamarca & 15 & 8 & 53,3 & 6 & 2 & 33,3 & 21 & 10 & 47,6 \\
\hline Chaco & 36 & 4 & 11,1 & 8 & 1 & 12,5 & 44 & 5 & 11,4 \\
\hline Chubut & 10 & 5 & 50,0 & 4 & 2 & 50,0 & 14 & 7 & 50,0 \\
\hline $\mathrm{CABA}$ & 29 & 3 & 10,3 & 44 & 5 & 11,4 & 73 & 8 & 11,0 \\
\hline Córdoba & 59 & 5 & 8,5 & 74 & 10 & 13,5 & 133 & 15 & 11,3 \\
\hline Corrientes & 26 & 4 & 15,4 & 7 & 0 & 0,0 & 33 & 4 & 12,1 \\
\hline Entre Ríos & 41 & 5 & 12,2 & 37 & 4 & 10,8 & 78 & 9 & 11,5 \\
\hline Formosa & 26 & 2 & 7,7 & 5 & 0 & 0,0 & 31 & 2 & 6,5 \\
\hline Jujuy & 15 & 7 & 46,7 & 2 & 0 & 0,0 & 17 & 7 & 41,2 \\
\hline La Pampa & 10 & 0 & 0,0 & 2 & 0 & 0,0 & 12 & 0 & 0,0 \\
\hline La Rioja & 32 & 2 & 6,3 & 1 & 0 & 0,0 & 33 & 2 & 6,1 \\
\hline Mendoza & 22 & 5 & 22,7 & 30 & 5 & 16,7 & 52 & 10 & 19,2 \\
\hline Misiones & 19 & 0 & 0,0 & 14 & 5 & 35,7 & 33 & 5 & 15,2 \\
\hline Neuquén & 20 & 0 & 0,0 & 3 & 2 & 66,7 & 23 & 2 & 8,7 \\
\hline Río Negro & 10 & 0 & 0,0 & 2 & 1 & 50,0 & 12 & 1 & 8,3 \\
\hline Salta & 49 & 4 & 8,2 & 15 & 4 & 26,7 & 64 & 8 & 12,5 \\
\hline San Juan & 9 & 1 & 11,1 & 9 & 1 & 11,1 & 18 & 2 & 11,1 \\
\hline San Luis & 2 & 1 & 50,0 & 1 & 0 & 0,0 & 3 & 1 & 33,3 \\
\hline Santa Cruz & 3 & 1 & 33,3 & 1 & 1 & 100,0 & 4 & 2 & 50,0 \\
\hline Santa Fe & 65 & 9 & 13,8 & 49 & 4 & 8,2 & 114 & 13 & 11,4 \\
\hline Santiago del Estero & 33 & 1 & 3,0 & 17 & 1 & 5,9 & 50 & 2 & 4,0 \\
\hline Tierra del Fuego & 3 & 2 & 66,7 & 1 & 0 & 0,0 & 4 & 2 & 50,0 \\
\hline Tucumán & 24 & 5 & 20,8 & 23 & 3 & 13,0 & 47 & 8 & 17,0 \\
\hline Total & 802 & 121 & 15,1 & 492 & 77 & 15,7 & 1294 & 198 & 15,3 \\
\hline
\end{tabular}

Tabla 4. Unidades que ofertan profesorados en lenguas extranjeras (año 2018).

extensiones áulicas que dependen de una unidad mayor. Se puede observar que en 198 IFD - el 15,3\% del total - se ofrecen profesorados de lenguas extranjeras, sin observarse casi diferencias en el promedio si se considera al sector público y al privado (Ver Tabla 4). Las diferencias entre jurisdicciones son relevantes, sin embargo, es sumamente llamativo que algunas tengan Fuente: Elaboración propia en base a datos del mapa de oferta de formación de docentes del INFD y los sitios web de las instituciones. 
Tabla 5. Apertura de profesorados de lenguas extranjeras según períodos históricos. Fuente: Elaboración propia en base a datos aportados por los propios IFD.

\begin{tabular}{l|c|c|c|c}
\hline Años & $\begin{array}{c}\text { Cantidad } \\
\text { profesorados }\end{array}$ & Públicos & Privados & \% del período \\
\hline $1904-1959$ & 12 & 9 & 3 & $\mathbf{5 , 8}$ \\
\hline $1960-1975$ & 29 & 17 & 12 & $\mathbf{1 3 , 9}$ \\
\hline $1976-1983$ & 14 & 7 & 7 & 6,7 \\
\hline $1984-1989$ & 18 & 12 & 6 & $\mathbf{8 , 7}$ \\
\hline $1990-1999$ & 37 & 18 & 19 & $\mathbf{1 7 , 8}$ \\
\hline $2000-2002$ & 20 & 10 & 10 & $\mathbf{9 , 6}$ \\
\hline $2003-2015$ & 74 & 54 & 20 & $\mathbf{3 5 , 6}$ \\
\hline $2016-p r e s e n t e$ & 4 & 4 & 0 & $\mathbf{1 , 9}$ \\
\hline Totales & $\mathbf{2 0 8}$ & $\mathbf{1 3 1}$ & $\mathbf{7 7}$ \\
\hline
\end{tabular}

tales como Chaco, Córdoba, Corrientes, Entre Ríos, Formosa, La Rioja, Neuquén, Río Negro, Salta, San Juan, Santa Fe y Santiago del Estero. Tales discrepancias en relación al sistema escapan a los alcances del presente artículo.

\section{La expansión del sistema}

¿Cómo fue el desarrollo histórico de la oferta de carreras de profesorado en lenguas extranjeras? De las 223 carreras contamos con el dato de apertura de 208. La Tabla 5 muestra el desarrollo histórico de los profesorados de lenguas extranjeras que, de alguna manera, es casi un sinónimo de los profesorados de inglés. De 1976 al presente hemos organizado la apertura de estas carreras en los IFD en etapas temporales que dan cuenta del período de duración de los gobiernos nacionales. Agrupamos al de Néstor Kirchner y el de Cristina Fernández en tanto entendemos una continuidad en las políticas educativas llevadas a cabo por ambos gobiernos.

La primera carrera de profesorado en lenguas extranjeras se crea en 1904 en la ciudad de Buenos Aires. Pero es destacable que desde 1976 al presente es decir en tan solo 43 años - se abre el 73,6 \% de las carreras de lenguas extranjeras relevadas, lo que pone de manifiesto un proceso de importantes dimensiones que se ha llevado a cabo en gran parte durante el último proceso democrático. Es importante señalar que durante el gobierno de Carlos Menem (1989-1999) se transfieren los profesorados a las provincias, por lo que les 
cabe a éstas el planeamiento, creación y sostenimiento presupuestario de los profesorados que comienzan a funcionar a partir de ese período, a los que se suman aquellos provinciales preexistentes (Ley 24.049 del año 1992). No es menor entonces el dato que en el período 2003-2015 se produce la apertura de 74 carreras de lenguas extranjeras: un nada desdeñable 35,6\% del total en lo que va del siglo XXI. Ya casi como un lugar común en este artículo, la mayoría de ellos son profesorados de inglés. Dada esta configuración que favorece marcadamente al inglés, se podría sospechar que desde el estado nacional hubo políticas que coordinaron la acción de las provincias para que así fuese, en función de un principio claro: el inglés debía ser priorizado por sobre las otras lenguas. ¿Sucedió efectivamente de esta forma?

\section{¿Dos lógicas diferentes de expansión de la oferta de formación docente?}

¿Cómo fue el proceso de expansión de estos profesorados? ¿Siguieron algún tipo de lógica estatal? En un estudio realizado por Diker y Terigi en el año 1994, ambas pedagogas observaban que los profesorados que no formaban docentes para los niveles primario e inicial se concentraban en los grandes centros urbanos del país. Las autoras señalaban además que «cuando hacemos constar la existencia de un establecimiento que ofrece carreras de formación docente para el nivel medio, se trata de una o dos carreras, tres a lo sumo. Son muy pocos los institutos que (como el INES Joaquín V. González de la Capital Federal) tienen un espectro amplio de carreras y están en condiciones de cubrir la oferta curricular de la escuela media» (Diker y Terigi, 1994:64).

Hoy la realidad parece reflejar un cuadro de situación sugestivamente diferente. En la actualidad, si observamos cómo se combinan los profesorados de idiomas extranjeros con otros profesorados, podemos apreciar que las instituciones que tienen tres o más profesorados son un $53,5 \%$ de total de los IFD que cuentan con inglés u otra lengua extranjera entre su oferta (ver Tabla 6). El porcentaje se eleva al $72,7 \%$ en el sector público, mientras que el sector privado concentra su oferta en instituciones que brindan solo un profesorado de lenguas extranjeras $(35,9 \%)$ o combinado con uno o dos profesorados de otros niveles o disciplinas (41\%). 


\begin{tabular}{lcccccc}
\hline Tipos de oferta & Pública & $\%$ & Privada & $\%$ & Total & $\%$ \\
\hline Idioma únicamente & 5 & 4,1 & 28 & 35,9 & 33 & 16,7 \\
\hline Idioma $+1 / 2$ profesorados & 28 & 23,1 & 32 & 41,0 & 60 & 30,3 \\
\hline Idioma $+3 / 4$ profesorados & 29 & 24,0 & 10 & 12,8 & 40 & 20,2 \\
\hline Idioma $+5 / 6$ profesorados & 31 & 25,6 & 3 & 3,8 & 34 & 17,2 \\
\hline Idioma $+7 / 8$ profesorados & 21 & 17,4 & 2 & 2,6 & 23 & 11,6 \\
\hline Idioma +9 profesorados o más & 7 & 5,8 & 2 & 2,6 & 9 & 4,5 \\
\hline Total & 121 & 100,0 & 78 & 100,0 & 198 & 100,0 \\
\hline
\end{tabular}

Tabla 6. Tipo de oferta de los IFD que cuentan con profesorados de idiomas extranjeros. Fuente: Elaboración propia en base al mapa de oferta de formación de docentes del INFD y los sitios web de las instituciones.
Si se cruza la información de la Tabla 6 con la población de los municipios en los que se encuentran los profesorados, se puede profundizar el análisis del proceso de expansión de la formación de profesores de lenguas extranjeras. A través de la Tabla 7 se corrobora algo ya observado por Diker y Terigi (1994) en tanto que el sector privado se extendió principalmente hacia municipios de más de 100000 habitantes. Mayoritariamente se caracterizan por ofertar solo el de lengua extranjera conviviendo con uno o dos profesorados más, generalmente de nivel primario y/o inicial.

Sin embargo, y en relación inversamente proporcional a lo señalado por las mencionadas pedagogas, en la actualidad el sector público tiene presencia en municipios de diversa cantidad de habitantes, contando con profesorados de idiomas extranjeros aun en distritos con menos de 50000 o 25000 habitantes. Además, estos profesorados públicos que han sido creados en el último cuarto de siglo se combinan en muchos casos con más de cinco carreras de otros niveles o disciplinas Ilegando en algunos casos a más de diez en distritos con menos de 100000 habitantes.

\section{El ingreso y la matrícula en el proceso de expansión reciente}

¿Cómo se han comportado el ingreso, el egreso y la matrícula en el marco de la gran expansión de los últimos años? Contamos con datos que representan el período 2011-2017, una serie relativamente corta para extraer conclusiones definitivas. Este es un ciclo de gran apertura de profesorados en tanto que la información disponible expresa un aumento de 170 a 198 


\begin{tabular}{|c|c|c|c|c|c|c|c|c|c|c|c|c|}
\hline & \multicolumn{2}{|c|}{$\begin{array}{l}0-25000 \\
\text { hab. }\end{array}$} & \multicolumn{2}{|c|}{$\begin{array}{c}25001-50000 \\
\text { hab. }\end{array}$} & \multicolumn{2}{|c|}{$\begin{array}{c}50001-100000 \\
\text { hab. }\end{array}$} & \multicolumn{2}{|c|}{$\begin{array}{c}100001-250000 \\
\text { hab. }\end{array}$} & \multicolumn{2}{|c|}{$\begin{array}{c}250001-500000 \\
\text { hab. }\end{array}$} & \multicolumn{2}{|c|}{$\begin{array}{c}\text { Más de } 500000 \\
\text { hab. }\end{array}$} \\
\hline & Público & Privado & Público & Privado & Público & Privado & Público & Privado & Público & Privado & Público & Privado \\
\hline $\begin{array}{l}\text { Idioma } \\
\text { únicamente }\end{array}$ & 2 & 0 & 0 & 0 & 0 & 1 & 0 & 3 & 0 & 9 & 3 & 15 \\
\hline $\begin{array}{l}\text { Idioma }+1 / 2 \\
\text { profesorados }\end{array}$ & 7 & 0 & 6 & 3 & 3 & 2 & 7 & 9 & 3 & 9 & 2 & 9 \\
\hline $\begin{array}{l}\text { Idioma }+3 / 4 \\
\text { profesorados }\end{array}$ & 4 & 0 & 5 & 1 & 9 & 2 & 4 & 3 & 4 & 4 & 3 & 1 \\
\hline $\begin{array}{l}\text { Idioma }+5 / 6 \\
\text { profesorados }\end{array}$ & 6 & 0 & 9 & 0 & 3 & 0 & 4 & 0 & 3 & 1 & 6 & 2 \\
\hline $\begin{array}{l}\text { Idioma }+7 / 8 \\
\text { profesorados }\end{array}$ & 3 & 0 & 2 & 0 & 7 & 0 & 4 & 0 & 3 & 1 & 2 & 1 \\
\hline $\begin{array}{l}\text { Idioma + } 9 \\
\text { profesorados } \\
\text { o más }\end{array}$ & 0 & 0 & 1 & 0 & 3 & 0 & 2 & 0 & 0 & 1 & 1 & 1 \\
\hline TOTAL & 22 & 0 & 23 & 4 & 25 & 5 & 21 & 15 & 13 & 25 & 17 & 29 \\
\hline
\end{tabular}

unidades con carreras de profesorado de lenguas extranjeras, es decir un incremento del $16,4 \%$. Si se consideran los años de inicio y finalización de la serie analizada, los ingresantes y los egresados aumentaron - a pesar de ciertas irregularidades a lo largo de los años - un 22\% (de 8430 a 10284 ingresantes) y 32,6\% respectivamente (de 1483 a 1966 egresados). Por su parte, la matrícula parece mostrar un sostenimiento mayor de las trayectorias de los estudiantes en tanto durante el período crece de manera bastante sostenida un 30,67\% (de 24139 a 31541 estudiantes matriculados). Los datos analizados expresan una apreciable expansión en dicho período que se manifiesta en un incremento relevante de unidades ofertantes de profesorados de lenguas extranjeras y, también, de las matrículas, ingresantes y egresados.

\section{La cuestión de la baja terminalidad}

Este importante crecimiento de la oferta de carreras de profesorados terciarios de lenguas extranjeras que se refleja en el aumento de los ingresantes, egresados y sostenimiento de la matrícula contrasta con la baja terminali-

Tabla 7. Tipo de oferta de los IFD que cuentan con profesorados de idiomas extranjeros y cantidad de habitantes de los municipios en los que se ubican. Fuente: Elaboración propia en base al mapa de oferta de formación de docentes del INFD y los sitios web de las instituciones. Los datos poblacionales han sido extraídos del Censo 2010. 
Gráfico I. Ingresantes, egresados y matrícula de los profesorados de lenguas extranjeras (2011-2016).

Fuente: Elaboración propia en base a datos del INFD.

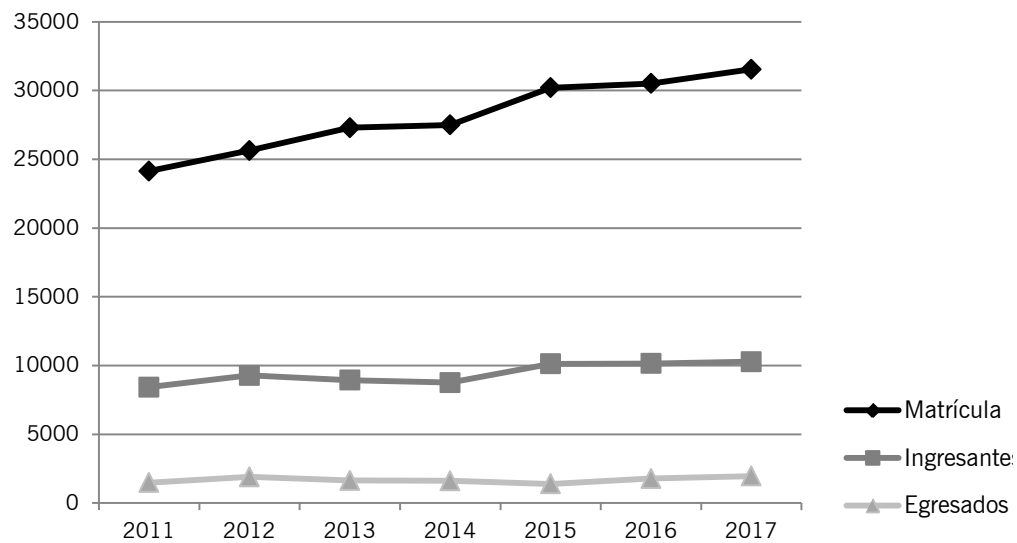

dad. El promedio nacional de egresados por unidad en el año 2011 es de 8,7 mientras que en 2017 el guarismo aumenta a 9,9 en el marco de un incremento de 28 nuevos IFD ofertando profesorados de lenguas extranjeras. Si se focaliza el análisis en las provincias se observa que ninguna tiene aumentos relevantes a excepción de Misiones, con un llamativo incremento que pasa de 5 a 254 egresados entre ambos años, la mayoría de lengua portuguesa. Carecemos de datos para dar cuenta de este importante crecimiento, que podría encontrar su origen en una deficiente recolección de la información por parte de las agencias estatales responsables. Sin embargo, parte de este aumento podría deberse a la influencia de la Ley 26.468 de Promoción del Portugués del año 2009. Dicha ley establece en su artículo 1 que para las provincias fronterizas con Brasil la inclusión del portugués es obligatoria en el nivel primario y secundario, mientras que para el resto de las jurisdicciones provinciales solo es obligatoria su inclusión en el nivel secundario. La realidad de proximidad geográfica, cultural y comercial con el Brasil podría explicar parcialmente tal crecimiento. Asimismo, tal como señala Bein (2012), los alcances de esta ley han sido muy limitados, ya que solo se ha logrado el carácter obligatorio de un taller optativo de portugués de al menos un año de duración y solo en la Orientación en Lenguas de la nueva escuela secundaria. 


\begin{tabular}{|c|c|c|c|c|c|c|}
\hline \multirow[b]{2}{*}{ Jurisdicción } & \multicolumn{3}{|c|}{2011} & \multicolumn{3}{|c|}{2017} \\
\hline & Egresados & Total unidades & $\begin{array}{c}\text { Promedio egresados } \\
\text { por unidad }\end{array}$ & Egresados & Total unidades & $\begin{array}{l}\text { Promedio } \\
\text { egresados por } \\
\text { unidad }\end{array}$ \\
\hline Buenos Aires & 618 & 65 & 9,5 & 743 & 73 & 10,2 \\
\hline Catamarca & 51 & 7 & 7,3 & 34 & 10 & 3,4 \\
\hline Chaco & 32 & 3 & 10,7 & 18 & 5 & 3,6 \\
\hline Chubut & 5 & 5 & 1,0 & 23 & 7 & 3,3 \\
\hline CABA & 159 & 9 & 17,7 & 190 & 8 & 23,8 \\
\hline Córdoba & 48 & 11 & 4,4 & 118 & 15 & 7,9 \\
\hline Corrientes & 29 & 3 & 9,7 & 43 & 4 & 10,8 \\
\hline Entre Ríos & 18 & 7 & 2,6 & 43 & 9 & 4,8 \\
\hline Formosa & 4 & 1 & 4,0 & 9 & 2 & 4,5 \\
\hline Jujuy & 13 & 7 & 1,9 & 41 & 7 & 5,9 \\
\hline La Rioja & 11 & 2 & 5,5 & 19 & 2 & 9,5 \\
\hline Mendoza & 36 & 9 & 4,0 & 80 & 10 & 8,0 \\
\hline Misiones & 5 & 5 & 1,0 & 254 & 5 & 51 \\
\hline Neuquén & 11 & 2 & 5,5 & 17 & 2 & 8,5 \\
\hline Río Negro & 72 & 1 & 72 & 9 & 1 & 9,0 \\
\hline Salta & 11 & 7 & 1,6 & 76 & 8 & 9,5 \\
\hline San Juan & 2 & 1 & 2,0 & 14 & 2 & 7,0 \\
\hline San Luis & 256 & 1 & 256 & 29 & 1 & 29 \\
\hline Santa Cruz & 1 & 2 & 0,5 & 11 & 2 & 5,5 \\
\hline Santa Fe & 39 & 12 & 3,3 & 83 & 13 & 6,4 \\
\hline Santiago del Estero & 19 & 2 & 9,5 & 11 & 2 & 5,5 \\
\hline Tierra del Fuego & 0 & 2 & 0,0 & 5 & 2 & 2,5 \\
\hline Tucumán & 43 & 6 & 7,2 & 96 & 8 & 12,0 \\
\hline Total & 1483 & 170 & 8,7 & 1966 & 198 & 9,9 \\
\hline
\end{tabular}

En función de los datos presentados, no podemos escapar al hecho de que la terminalidad es baja, sobre todo si tomamos en consideración la expansión reciente en la oferta de carreras de profesorados que hemos analizado. Sin embargo, y no a modo de excusa, sino como enmarcamiento dentro de una problemática que excede a la de la formación de docentes terciarios en lenguas extranjeras, la baja terminalidad no es una cuestión excluyente de este campo, sino que caracteriza al sistema de formación docente en general y al sistema de educación superior en su conjunto. Comenzar a
Tabla 8. Promedio egresados por unidades de IFD (años 2011 y 2017).

Fuente: Elaboración propia en base a datos del INFD. 
dar cuenta de ella significa elegir pensarla como una cuestión de agenda educativa relevante.

\section{Egresados por lenguas}

El último aspecto que analizaremos es el los egresados por cada una de las lenguas extranjeras. Al respecto, se percibe la preeminencia de los graduados de los profesorados de lengua inglesa sobre los cuatro idiomas restantes. Esto no es Ilamativo pues está en relación directa con la enorme diferencia en la cantidad de profesorados disponibles para cada una de las lenguas extranjeras. El año 2017 constituye un buen ejemplo para comparar la cantidad de egresados de los profesorados de IFD en tanto que de los de inglés titularon a un total de 1655 profesores (84.18\%) 29 los de francés (1.47\%), 9 los de alemán $(0,45 \%), 8$ los de italiano $(0,40 \%)$ y 265 los de portugués $(13,47 \%$ ) (ver Gráfico II). Cabe señalar que el incremento que se observa en el resto de las lenguas extranjeras entre el año 2016 y el 2017 se explica en la diferencia interanual de 18 a 265 egresados de lengua portuguesa.

En este caso, los números son sumamente elocuentes acerca de la contundencia de tal diferencia, por lo que la abordaremos en la próxima sección como una problemática y un eje traccionador en sí mismo.

\section{Expansión de la formación docente en lenguas extranjeras con hegemonía del inglés: ¿Una política por defecto?}

Los números compartidos muestran la gran expansión del sistema formador terciario de profesores de lenguas extranjeras, proceso que se concreta particularmente desde el retorno de la democracia hasta el presente. Un enorme crecimiento que acontece en el marco de la expansión del sistema formador terciario en su totalidad, tal como lo muestran diversos trabajos con base estadística realizados desde mediados de la década del noventa (Diker y Terigi, 1994, Davini, 2005, Aguerrondo y Vezub, 2011, INFD, 


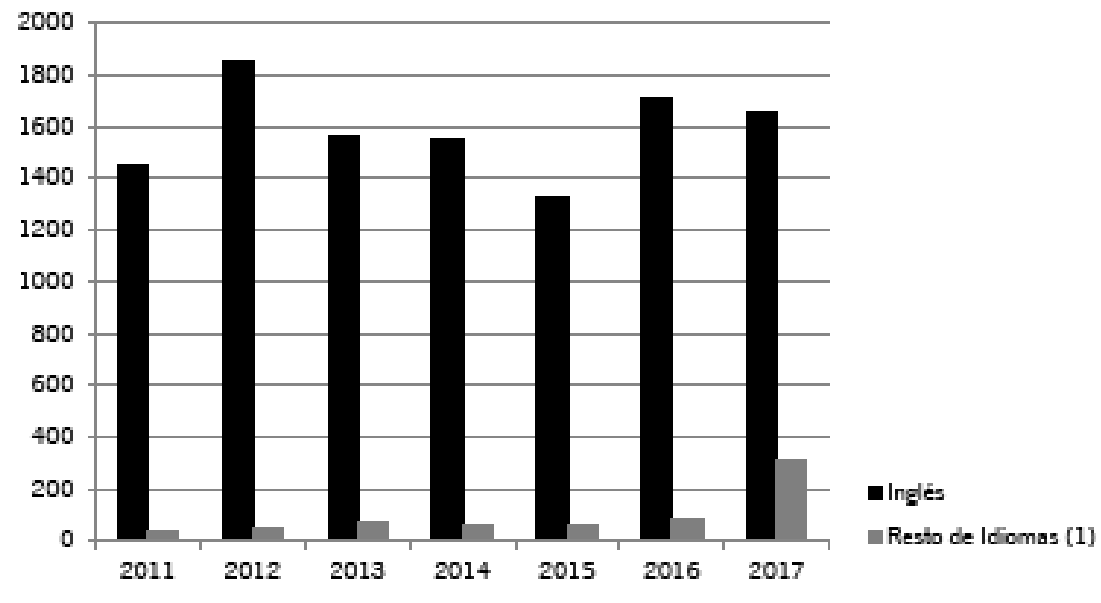

2014). También hemos observado que tal fenómeno se caracteriza por un incremento de la matrícula, los egresados y los ingresantes con la contrapartida de una muy baja terminalidad.

No conocemos trabajos recientes que revelen las necesidades de profesores de lenguas extranjeras titulados que precisa el sistema educativo. Sin embargo, el interesante mapeo que realizó el CIPPEC en el año 2007 mostraba cómo las distintas provincias fueron avanzando en sus políticas para ampliar la enseñanza de idiomas en los distintos niveles educativos. Además, el mencionado estudio reflejaba que para el año 2007 en el sistema educativo estatal «el $30 \%$ de los docentes que enseñan una lengua extranjera en el nivel primario (EGB 1 y 2 ) y en el secundario básico (EGB 3 ) no tiene formación en lengua extranjera. Esta proporción desciende al 25\% para el secundario (Polimodal)» (CIPPEC, 2007:27).

A continuación, nos detendremos en una característica que tiñe a la formación de profesores terciarios de lenguas extranjeras: el claro predominio del inglés por sobre las otras lenguas extranjeras. ¿Es esta hegemonía el producto de lineamientos y definiciones políticas explícitamente planificadas por las gestiones educativas nacionales y provinciales? Hemos analizado en otros trabajos las normativas educativas y materiales curriculares del estado nacional vinculadas con las lenguas extranjeras desde mediados del siglo
Gráfico II. Egresados de los profesorados de inglés y del resto de las lenguas extranjeras (2011-2017).

Fuente: Elaboración propia en base a datos del INFD. 
XIX hasta la actualidad en relación con la educación básica (Mórtola y Montserrat, 2018). Tanto las normas nacionales como muchos documentos que emanaron desde el estado central oscilan entre igualarlas en una secuencia que enuncia al inglés, francés, alemán, italiano y portugués en su totalidad o en gran parte de ellas 0 , alternativamente, nos encontramos con textos en los que aparece alguna de ellas destacada por sobre las otras. Si focalizamos nuestra atención en el período 2003-2015 se observa una igualación teórica y retórica de las cinco lenguas en la mayor parte de los documentos que fueron aprobados en el marco del Consejo Federal de Educación como ámbito de concertación de las políticas educativas del país.

Consideramos, por lo tanto, que la potencia del proceso que hace hegemónico al inglés en el sistema formador docente no puede explicarse si no se analiza la interrelación entre el inglés como lengua de comunicación internacional, las demandas sociales que expresan una aspiración por una movilidad social ascendente y las ideologías del lenguaje, tres fenómenos complejos que llevan al menos un siglo de construcción constante a la luz de transformaciones globales. Desde nuestra perspectiva, el inglés se constituyó en una aspiración social con un carácter marcadamente instrumental/laboral respecto del aprendizaje de una lengua extranjera.

Ahora bien, ¿qué significación pueden tener las aspiraciones sociales para los decisores de políticas públicas? Hart (2016) sostiene que las aspiraciones sociales pueden establecer el curso de la acción pública, la política, la inversión, la regulación y la legislación. Ciertas aspiraciones sociales que se convierten en hegemónicas obtienen en muchas ocasiones una poderosa capacidad de influencia y persuasión sobre los diversos actores sociales que participan de ellas. Es entonces que los mismos agentes del estado, desde los mismos técnicos responsables de la generación de políticas hasta los mismos docentes en las escuelas, poseen ideologías del lenguaje de las que frecuentemente no se encuentran conscientes y que influyen sobre sus decisiones.

Es entonces que comenzar a comprender cómo operan las aspiraciones sociales implica también dar cuenta del juego de dinámicas que las constituyen. Una forma de aproximarnos a ellas es a través del concepto de ideología del lenguaje (Woolard, 2009) que expresa a aquellas representaciones del 
sentido común que las personas poseen acerca de las lenguas, las que son tanto colectivas como frecuentemente irreflexivas. Como tales, se generan a partir de experiencias subjetivas que luego son generalizadas y ampliamente compartidas. Formar parte de una determinada sociedad y un sector social específico implica encontrarse inmerso en un océano de ideologías del lenguaje, a menudo contrapuestas, contradictorias y no explicitadas. En este sentido, una de las creencias generalizadas acerca del inglés es que dominarlo posibilita el acceso a mejores oportunidades laborales, lo que en palabras del sentido común se expresa en «el inglés abre puertas». Como sugiere Seargeant (2009) las creencias acerca del lenguaje son centrales a sus políticas y, como tales, rara vez son solamente acerca de la lengua en sí misma - en este caso el inglés - sino que también involucran a las asociaciones que una sociedad dada genera con la lengua y los usos de la misma. Estas pueden devenir aspiraciones sociales que traccionen al estado y al mercado reduciendo los márgenes de direccionamiento real de las políticas. ¿Cómo podrían interpretarse estos posicionamientos teóricos con relación a la formación docente terciaria en lenguas extranjeras en nuestro país? Creemos que esta es el producto de un complejo entramado de dinámicas sociales en el que han jugado con potencia el inglés como lengua internacional y las aspiraciones sociales por hacerse de él. El inglés porta elementos ideológicos, que aunque diferenciados en su sofisticación e importancia, se encuentran entretejidos alrededor de un mínimo común denominador: no encontrarse en posesión de la lengua significa correr el riesgo de quedarse por fuera de la carrera por la movilidad social. Tales demandas sociales se vieron entonces reflejadas en la expansión del sistema formador de docentes en lenguas extranjeras, con las particularidades propias de cada jurisdicción. En síntesis, el estado reaccionó a las demandas sociales, y bien vale recalcar este término, más que planificó, al momento de enfrentarse a las ideologías del lenguaje acerca del rol del inglés en nuestra sociedad.

Finalmente, no relevar, estudiar y analizar estas dinámicas podría implicar por default un rol del estado cada vez más disminuido e irrelevante, al decir de Fernández Vítores (2009), una «política por defecto» que implícitamente contará con un solo claro ganador: el inglés 


\section{Conclusiones}

A lo largo de este artículo, hemos llevado a cabo un análisis del sistema de formación de docentes terciarios en lenguas extranjeras. Puede señalarse en primer lugar que el plurilingüismo como paradigma académico predominante parece encontrarse en la encrucijada si tomamos en consideración la magnitud de las cifras que constituyen la hegemonía del inglés en este subsistema. Recordemos: el $86,5 \%$ de los profesorados terciarios del país son en inglés.

Esta configuración no puede comprenderse si no se la interpreta a la luz de la tríada conformadas por aspiraciones sociales, las motivaciones vinculadas con una movilidad social ascendente y las ideologías del lenguaje predominantes. Estos tres factores, entendidos como complejos fenómenos sociales, interactúan entre sí y son sumamente sensibles a factores tanto locales como globales. En una era de interconexión ubicua, las sociedades interactúan configurando su propia versión idiosincrática de "glocalización». Esta implica la simultaneidad - la co-presencia - tanto de tendencias hacia la universalización como hacia la particularización (Robertson, 1997). En este sentido, la hegemonía del inglés y su influencia sobre nuestro sistema educativo no puede ser comprendida en profundidad si no se toman en consideración las dinámicas globales y cómo estas son reinterpretadas localmente en un proceso que se construye en forma simultánea.

También hemos visto cómo el sistema ha crecido sostenida y marcadamente en los últimos 40 años. A diferencia de etapas anteriores, en los últimos quince años la lógica de tal crecimiento, al menos en parte, fue la del acercamiento paulatino a núcleos poblacionales más pequeños. Aunque no explicitado a través de las políticas, estas parecerían expresar en la práctica un anhelo democrático de parte del estado de llegar hasta donde no se había llegado antes. Finalmente, la baja terminalidad general impacta negativamente sobre el número de docentes terciarios necesarios en cada una de las jurisdicciones, lo que podría implicar en la práctica circunscribir la enseñanza de una lengua extranjera solamente al inglés, dado su marcado predominio. 
Hemos partido entonces de un principio simple: realizar un mapeo general de aquello que se encuentra actualmente configurado e intentar comprender por qué razones se ha constituido como tal a la búsqueda de regularidades y fenómenos que aun requieren, obviamente, de estudios futuros. Esperamos entonces que este artículo contribuya a un debate necesario en el campo de la formación de docentes terciarios en lenguas extranjeras y que tanto los datos abordados como las hipótesis provisionales propuestas puedan contribuir a éste.

\section{Referencias bibliográficas}

Aguerrondo, I. y Vezub, L. (2011). Las instituciones terciarias de formación docente en Argentina. Condiciones institucionales para el liderazgo pedagógico. Educar, Vol. 47/2, pp. 211-235. Recuperado de https://ddd.uab.cat/pub/educar/0211819Xv47n2/educar_a2011v47n2p211.pdf

Bein, R. (2012). Argentinos: esencialmente europeos. Quaderna, 1/12, pp. 1-17. Recuperado de http://quaderna.org/wp-content/uploads/2012/12/BEIN-argentinos-esencialmente.pdf

CIPPEC (2007). La enseñanza universal de lenguas extranjeras. Buenos Aires: CIPPEC

Davini, M. C. (2005). Estudio de la calidad y la cantidad de oferta de la formación docente, investigación y capacitación en Argentina. Buenos Aires: Ministerio de Educación

Diker, G. y Terigi, F. (1994). Panorama de la formación docente en Argentina. Buenos Aires: Ministerio de Educación/OEI
Fernández Vítores, D. (2009). El inglés en Europa: origen y consolidación de una lengua franca. Odisea, No 10 , pp. 57-69

Hart, C. (2016). Aspirations, education and social justice: applying Sen and Bourdieu. Bloomsbury. Vol. 4, pp. 65-78. Recuperado de https://www.researchgate.net/publication/263684363_Aspirations_education_and_social_justice_applying_Sen_and_Bourdieu Instituto Nacional de Formación Docente (2014). La formación docente en cifras. Argentina 2014. Buenos Aires: Ministerio de Educación

Mórtola, G. y Montserrat, M. (2018). La enseñanza del inglés para las grandes mayorías nacionales en Argentina. Revista Digital de Políticas Lingüísticas. Año 10, Vol. 10, pp. 167-191. Disponible en: https://revistas. unc.edu.ar/index.php/RDPL/article/view/22198/21804 
Mórtola, G. y Montserrat, M. (2019). El sistema universitario argentino y las lenguas extranjeras: perdidos en el laberinto de la hegemonía del inglés, las ideologías del lenguaje y la jerarquización de las titulaciones. Revista Ucronías (en prensa)

Robertson, R. (1997). The conceptual promise of glocalization: commonality and diversity. Glocalogue, issue 4 Recuperado de http://artefact.mi2.hr/_a04/lang_en/ theory_robertson_en.htm

Seargeant. P. (2012). The politics and policies of global English. EnHewings, A. and Tagg, C. (eds), The Politics of English: conflict, competition, pp. 5-30. Routledge: The Open University.
Woolard, K. (2009). Language Ideologies: Issues and Approaches. PragmaticsVol 2, (3), pp. 235 -249. Recuperado de https://journals.linguisticsociety.org/elanguage/pragmatics/article/download/225/225-1833-1PB.pdf

\section{Sitios ministeriales consultados}

Mapa de Institutos de Argentina (INFD): https://mapa. infd.edu.ar/

Secretaría de Políticas Universitarias. Guías de Carreras Universitarias: http://ofertasgrado.siu.edu.ar/ 